\title{
Energy of wave and solutions of C-Holm equation under Lagrangian point of view
}

\author{
${ }^{1}$ Sh Hajrulla, L Bezati ${ }^{2}$ \& F Hoxha ${ }^{2}$ \\ ${ }^{1}$ University of Vlora, Albania, \\ University of Tirana, Albania ${ }^{2}$
}

\begin{abstract}
:
We deal with the Camassa-Holm equation $u_{t}-u_{x x t}+2 k u_{x}+3 u u_{x x}-u u_{x x x}=0$ possesses a global continuous semigroup of weak conservative solutions for initial data $\left.u\right|_{t=0}=\bar{u}$ in $H^{1}$. The result is obtained by introducing a coordinate transformation into Lagrangian coordinates. To characterize conservative solutions it is necessary to include the energy density given by the positive Radon measure $\mu$ with $\mu_{a c}=\left(u^{2}+u_{x}^{2}\right) d x$. The total energy is preserved by the solution.
\end{abstract}

Key words: weak solutions; Camassa-Holm equation; Radon measure; energy density; global existence of solution; total energy.

\section{Introduction}

In this paper, we reformulate the Camassa - Holm equation using a different set of variables and obtain a semilinear system of ordinary differential equations, as Bressan and Constantin [1]. The Cauchy problem for the Camassa - Holm equation [4], [5].

$$
u_{t}-u_{x x t}+2 k u_{x}+3 u u_{x x}-u u_{x x x}=0,\left.\quad u\right|_{t=0}=\bar{u}
$$

has received considerable attention the last decade. With $k$ positive it models, see [2], [7], propagation of unidirectional gravitational waves in a shallow water approximation, with $u$ representing the fluid velocity. The Camassa-Holm equation has a bi-Hamiltonian structure and is completely integrable. It has infinitely many conserved quantities. In particular, for smooth solutions the quantities

$$
\int u d x, \quad \int\left(u^{2}+u_{x}^{2}\right) d x, \quad \int\left(u^{3}+u u_{x}^{2}\right) d x
$$

are all time independent.

In this article we consider the case $k=0$ on the real line, that is

$$
u_{t}-u_{x x t}+3 u u_{x}-2 u_{x} u_{x x}-u u_{x x x}=0
$$

and henceforth we refer to (1.3) as the Camassa - Holm equation. The equation can be rewritten as the following system

$$
\begin{gathered}
u_{t}+u u_{x}+P_{x}=0, \\
P-P_{x x}=u^{2}+\frac{1}{2} u_{x}^{2}
\end{gathered}
$$


A highly interesting property of the equation is that for a wide class of initial data the solution experiences wave breaking in finite time in the sense that the solution $u$ remains bounded pointwise while the spatial derivative $u_{x}$ become unbounded point wise. However, the $H^{1}$ norm of $u$ remains finite. More precisely, Constantin, Escher and Molinet $[8,10]$ showed the following result: If the initial data $\left.u\right|_{t=0}=\overline{u \in H^{1}(R)}$ and $\bar{m}:=\bar{u}-\bar{u}^{\prime \prime}$ is a positive Radon measure, then equation (1.3) has a unique global weak solution $u \in\left(C[0, T], H^{1}(R)\right)$, for any $T$ positive, with initial data $\bar{u}$. However, any solution with odd initial data $\bar{u}$ in $H^{3}(R)$ such that $\bar{u}_{x}(0)<0$ blows up in a finite time.

The problem how to extend the solution beyond wave breaking can nicely be illustrated by studying an explicit class of solutions. The Camassa -Holm equation possesses solutions, denoted (multi) peakons, of the form

$$
u(t, x)=\sum_{i=1}^{n} p_{i}(t) e^{-\left|x-q_{i}(t)\right|},
$$

where the $\left(p_{i}(t), q_{i}(t)\right)$ satisfy the explicit system of ordinary differential equations

$$
\dot{q}_{i}=\sum_{j=i}^{n} p_{j} e^{-\left|q_{i}-q_{j}\right|}, \quad \dot{q}_{i}=\sum_{j=1}^{n} p_{i} p_{j} \operatorname{sgn}\left(q_{i}-q_{j}\right) e^{-\left|q_{i}-q_{j}\right|}
$$

Observe that the solution (1.5) is not smooth even with continuous functions $\left(p_{i}(t), q_{i}(t)\right)$; one possible way to interpret (1.5) as a weak solution of (1.3) is to rewrite the equation (1.3) as

$$
u_{t}+\left(\frac{1}{2} u^{2}+\left(1-\partial_{x}^{2}\right)^{-1}\left(u^{2}+\frac{1}{2} u_{x}^{2}\right)\right)_{x}=0
$$

Peakons interact in a way similar to that of solutions of the Korteweg-de Vries (KdV) equation, and wave breaking may appear when at least two of the $q_{i}$ 's coincide. If all the $p_{i}(0)$ have the same sign, the peakons move in the same direction. Furthermore, in that case the solution experiences no wave breaking, and one has a global solution. Higher peakons move faster than the smaller ones, and when a higher peakon overtakes a smaller, there is an exchange of mass, but no wave breaking takes place. Furthermore, the $q_{i}(t)$ remain distinct. However, if some of $p_{i}(0)$ have opposite sign, wave breaking may incur, see, e.g., $[3,6]$.

For simplicity, consider the case with $n=2$ and one peakon $p_{1}(0)>0$ (moving to the right) and one antipeakon $p_{2}(0)<0$ (moving to the left). In the symmetric case $\left(p_{1}(0)=-p_{2}(0)\right.$ and $q_{1}(0)=$ $\left.-q_{2}(0)<0\right)$ the solution will vanish pointwise at the collision time $t^{*}$ when $q_{1}\left(t^{*}\right)=q_{2}\left(t^{*}\right)$, that is, $u\left(t^{*}, x\right)=0$ for all $x \in R$. Clearly, at least two scenarios are possible; one is to let the peakon and antipeakon "pass through" each other in a way that is consistent with the Camassa - Holm equation. In the first case the energy $\int\left(u^{2}+u_{x}^{2}\right) d x$ decreases to zero at $t^{*}$. Clearly, the well-posedness of the equation is a delicate matter in this case. The first solution could be denoted a dissipative solution, while the second one could be called conservative. Other solutions are also possible.

The problem of continuation beyond wave breaking was recently considered by Bressan and Constantin [4]. They reformulated the Camassa-Holm equation as a semilinear system of ordinary differential equations taking values in a Banach space. This formulation allowed them to continue the solution beyond collision time, giving a global conservative solution where the energy is conserved for almost all times.

Going back to the original function $u$, one obtains a global solution of the Camassa-Holm equation. The well-posedness, i.e.,the uniqueness and stability of the solution, is resolved as follows. In addition to the solution $\mathrm{u}$, one includes a family of non-negative Radon measures $\mu_{t}$ with density $u_{x}^{2} d x$ with respect to the Lebesgue measure. The pair $\left(u, \mu_{t}\right)$ constitutes a continuous semigroup, in particular, one has uniqueness and stability.

Very recently, Bressan and Fonte $[5,11]$ presented another approach to the Camassa-Holm equation. The flow map $\bar{u} \rightarrow u(t)$ is, as we have seen, neither a continuous map on $H^{1}$ nor on $L^{2}$. However, they introduced a new distance $J(u, v)$ with the property 
It satisfies

$$
c_{1}\|u-v\|_{L^{1}} \leq J(u, v) \leq c_{2}\|u-v\|_{H^{1}}
$$

$$
J(u(t), \bar{u}) \leq c_{3}|t|, \quad J(u(t), v(t)) \leq J(\bar{u}, \bar{v}) e^{c_{4}|t|}
$$

where $u(t), v(t)$ are solutions with initial data $\bar{u}, \bar{v}^{-}$respectively. The distance is introduced by first defining using the global, conservative solution described above. This enables them to construct conservative solutions for the Camassa-Holm equation which is continuous with respect to the distance $J$.

\section{Premilinary}

In this paper, we reformulate the equation using a different set of variables and obtain a semilinear system of ordinary differential equations, as Bressan and Constantin [1].

However, distinct variables from that simply corresponds to the transformation between Eulerian and Lagrangian coordinates.

Let $u=u(t, x)$ denote the solution, and $y(t, \xi)$ the corresponding characteristics, thus $y_{t}(t, \xi)=$ $u(t, y(t, \xi))$. Our new variables are $y(t, \xi)$,

$$
U(t, \xi)=u(t, y(t, \xi)), H(t, \xi)=\int_{-\infty}^{y(t, \xi)}\left(u^{2}+u_{x}^{2}\right) d x
$$

where $\mathrm{U}$ corresponds to the Lagrangian velocity while $\mathrm{H}$ could be interpreted as the Lagrangian cumulative energy distribution. The characteristics $q(\xi ; t)$ are defined as solutions of the equation

$$
q_{t}(\xi ; t)=m(q(\xi ; t), t)
$$

with the initial condition $q(\xi ; t)=\xi$. Let we consider the momentum $m=u-u_{x x}$ of the system and introduce the variable $p$ related direct to the momentum, as

$$
p(\xi ; t)=m\left(q(\xi ; t), \frac{\partial_{q}}{\partial_{\xi}}(\xi ; t)\right)
$$

Camassa shows the following system of partial differential equations

Furthermore, let

$$
\begin{array}{r}
q_{t}(\xi ; t)=\frac{1}{2} \int_{R} \exp (-|q(\xi ; t)-q(\mu ; t)|) p(\mu ; t) d \mu \\
q_{t}(\xi ; t)=\frac{1}{2} \int_{R} \operatorname{sgn}(\xi-\mu) \exp (-|q(\xi ; t)-q(\mu ; t)|) p(\mu ; t) d \mu
\end{array}
$$

$$
\begin{array}{r}
P(\xi ; t)=\frac{1}{2} \int_{R} \exp (-|q(\xi ; t)-q(\mu ; t)|)\left(U^{2} y_{\xi}+H_{\xi}\right) d \mu \\
Q(\xi ; t)=\frac{1}{2} \int_{R} \operatorname{sgn}(\xi-\mu) \exp (-|q(\xi ; t)-q(\mu ; t)|)\left(U^{2} y_{\xi}+H_{\xi}\right) d \mu
\end{array}
$$

Then we can show that the following system

$$
\left\{\begin{array}{c}
y_{t}=U \\
U_{t}=-Q \\
H_{t}=U^{3}-2 P U
\end{array}\right.
$$

is equivalent to the Camassa-Holm equation. 
Global existence of solutions of (1.7) is obtained starting from a contraction argument. As noted in [1], even if $H^{1}(R)$ is a natural space for the equation, there is no hope to obtain a group of solutions by only considering $H^{1}(R)$. Thus, we introduce the following space $D$, which characterizes the solutions in Eulerian coordinates.

Definition 2.1. The set $D$ is composed of all pairs $(u, \mu)$ such that $u$ belongs to $H^{1}(R)$ and $\mu$ is a positive finite Radon measure whose absolute continuous part, satisfies $\mu_{a c}=\left(u^{2}+u_{x}^{2}\right) d x$.

For a given $\xi$, can also be seen as the position of a particle evolving in the velocity field $u$, where $u$ is the solution of the Camassa-Holm equation. We are then working in Lagrangian coordinates.

The pairs $(u, \mu)$ such that $(u, \mu) \in D$ if $u \in H^{1}(R)$ and $\mu$ is a positive Radon measure whose absolutely continuous part satisfies $\mu_{a c}=\left(u^{2}+u_{x}^{2}\right) d x$. With three Lagrangian variables $(y, U, H)$ versus two Eulerian variables $(u, \mu)$, we define a group of transformations which acts on the Lagrangian variables and let the system of equations (1.7) invariant. Using this group, we are able to establish a bijection between the space of Eulerian variables and the space of Lagrangian variables when we identify variables that are invariant. This bijection allows us to transform the results obtained in the Lagrangian framework into the Eulerian framework .

In particular, and this constitutes the main result of this paper, we obtain a metric $d_{D}$ on $D$ and a continuous semi-group of solutions on $\left(D, d_{D}\right)$. The distance $d_{D}$ gives $D$ the structure of a complete metric space. This metric is compared with some more standard topologies, and we obtain that convergence in $H^{1}(R)$ implies convergence in $\left(D, d_{D}\right)$ which itself implies convergence in $L^{\infty}(R)$, Our main result states that for given initial data in $D$ there exists a unique weak solution of the Camassa-Holm equation. The associated measure $\mu(t)$ has constant total mass, i.e., $\mu(t)(R)=\mu(0)(R)$ for all $t$, which corresponds to the total energy of the system. This is the reason why our solutions are called conservative.

The method described can be studied in detail for multipeakons, see [12] for details. By suitably modifying the techniques described in this paper, the results can be extended to show global existence of conservative solutions for the generalized equation

$$
\left\{\begin{array}{c}
u_{t}+f(u)_{x}+P_{x}=U \\
P-P_{x x}=g(U)+\frac{1}{2} f^{\prime \prime}(u) u_{x}^{2}
\end{array}\right.
$$

where $f, g \in L^{\infty}(R)$. Observe that if $g(u)=k u+u^{2}$ and $f(u)=k u+\frac{u^{2}}{2}$, then (1.8) is the classical Camassa-Holm equation (1.1). See [13] for a recent proof of existence of dissipative solutions of (1.8). The details will be not described in this paper. Furthermore, the methods presented in this paper can be used to derive numerical methods that converge to conservative solutions.

\subsection{Global solutions in Lagrangian coordinates}

Assuming that $u$ is smooth, it is not hard to check that

$$
\left(u^{2}+u_{x}^{2}\right)_{t}+\left(u\left(u^{2}+u_{x}^{2}\right)\right)_{x}=\left(u^{3}-2 P u\right)_{x}
$$

Let us introduce the characteristics $y(t ; \xi)$ defined as the solutions of

$$
u_{t}(t ; \xi)=u(t, y(t, \xi))
$$

for a given $y(0 ; \xi)$. Equation (2.1) gives us information about the evolution of the amount of energy contained between two characteristics. Indeed, given $\xi_{1}, \xi_{2}$ in $R$, let $H(t)=\int_{y\left(t, \xi_{1}\right)}^{y\left(t, \xi_{2}\right)}\left(u^{2}+u_{x}^{2}\right) d x$ be the energy contained between the two characteristic curves $y\left(t, \xi_{1}\right)$ and $y\left(t, \xi_{2}\right)$. Then, using (2.1) and (2.2), we obtain 


$$
\frac{d H}{d t}=\left.\left(\left(u^{3}-2 P u\right)^{\circ} y\right)\right|_{\xi_{1}} ^{\xi_{2}}
$$

Solutions of the Camassa-Holm blow up when characteristics arising from different points collide. It is important to notice that we do not get shocks as the Camassa-Holm preserves the $H^{1}$ norm and therefore solutions remain continuous. However, it is not obvious how to continue the solution after collision time. It turns out that, when two characteristics collide, the energy contained between these two characteristics has a limit which can be computed from (2.3). As we will see, knowing this energy enables us to prolong the characteristics and thereby the solution, after collisions.

We now derive a system equivalent to (1.4). All the derivations in this section are formal and will be justified later. Let y still denote the characteristics. We introduce two other variables, the Lagrangian velocity and cumulative energy distribution, $U$ and $H$, defined as $U(t, \xi)=u(t, y(t \xi))$ and

$$
H(t)=\int_{-\infty}^{y(t, \xi)}\left(u^{2}+u_{x}^{2}\right) d x
$$

From the definition of the characteristics, it follows that

$$
U_{t}(t ; \xi)=u_{t}(t, y)+y_{t}(t, \xi) u_{x}(t, y)=-P_{x}^{\circ} y(t, \xi)
$$

This last term can be expressed uniquely in term of $U, y$, and $H$. From (1.4b), we obtain the following explicit expression for ,

$$
P(\xi, t)=\frac{1}{2} \int_{R} e^{|x-z|}\left(u^{2}(t, z)+\frac{1}{2} u_{x}^{2}(t, z)\right) d z
$$

Thus we have

$$
P_{x}^{\circ} y(t, \xi)=-\frac{1}{2} \int_{R} \operatorname{sgn}(y(t, \xi)-z) e^{-|y(t, \xi)-z|}\left(u^{2}(t, z)+\frac{1}{2} u_{x}^{2}(t, z)\right) d z
$$

Since $H_{\xi}=\left(u^{2}+u_{x}^{2}\right)^{\circ} y y_{\xi}$

$$
P_{x}^{\circ} y(t, \xi)=-\frac{1}{4} \int_{R} \operatorname{sgn}(y(\xi)-y(\mu)) \exp (-|y(\xi)-y(\mu)|)\left(U^{2} y_{\xi}+H_{\xi}\right) \mu d \mu
$$

where the $\mathrm{t}$ variable has been dropped to simplify the notation. Later we will prove that $\mathrm{y}$ is an increasing function for any fixed time t. If, for the moment, we take this for granted, then $P_{x}^{\circ} y$ is equivalent to $Q$ where

$$
\begin{aligned}
& Q(t, \xi)=-\frac{1}{4} \int_{R} \operatorname{sgn}(\xi-\mu) \exp (-\operatorname{sgn}(\xi-\mu)(y(\xi)-y(\mu)))\left(U^{2} y_{\xi}+H_{\xi}\right) \mu d \mu \\
& P(t, \xi)=-\frac{1}{4} \int_{R} \exp (-\operatorname{sgn}(\xi-\mu)(y(\xi)-y(\mu)))\left(U^{2} y_{\xi}+H_{\xi}\right) \mu d \mu
\end{aligned}
$$

Thus $P_{x}^{\circ} y$ and $P^{\circ} y$ can be replaced by equivalent expressions given by (2.8) and (2.9) which only depend on our new variables $U, H$, and $y$. We introduce yet another variable, $\zeta(t ; \xi)$, simply defined as $\zeta(t, \xi)=$ $y(t, \xi)-\xi$. It will turn out that $\zeta \in L^{\infty}(R)$. We now derive a new system of equations, formally equivalent to the Camassa-Holm equation. Equations (2.5), (2.3) and (2.2) give us 


$$
\left\{\begin{array}{c}
\zeta_{t}=U \\
U_{t}=-Q \\
H_{t}=U^{3}-2 P U
\end{array}\right.
$$

As we will see, the system (2.10) of ordinary differential equations for $(\zeta, U, H)$ from $[0 ; T]$ to $E$ is wellposed, where $E$ is Banach space to be defined in the next section. We have

$$
Q_{\xi}=-\frac{1}{2} H_{\xi}-\left(\frac{1}{2} U^{2}-P\right) y_{\xi} \quad \text { and } \quad P_{\xi}=Q y_{\xi}
$$

Hence, differentiating (2.10) yields

$$
\left\{\begin{array}{c}
\zeta_{\xi t}=U_{\xi}\left(\text { or } y_{\xi t}=U_{\xi}\right) \\
U_{\xi t}=\frac{1}{2} H_{\xi}-\left(\frac{1}{2} U^{2}-P\right) y_{\xi} \\
H_{\xi t}=-2 Q U y_{\xi}+\left(3 U^{2}-2 P\right) U_{\xi}
\end{array}\right.
$$

The system (2.12) is semilinear with respect to the variables $y_{\xi}, U_{\xi}$ and $H_{\xi}$.

\subsection{Existence and uniqueness of solutions of the equivalent system.}

In this section, we focus our attention on the system of equations (2.10) and prove, by a contraction argument, that it admits a unique solution. Let $\mathrm{V}$ be the Banach space defined by

$$
V=\left\{f \in C_{b}(R) \mid f_{\xi} \in L^{2}(R)\right\}
$$

where $C_{b}(R)=C(R) \cap L^{2}(R)$ and the norm of $V$ is given by $\|f\|_{V}=\|f\|_{L^{\infty}(R)}+\|f\|_{L^{2}(R)}$. Of course $H^{1}(R) \subset V$ but the converse is not true as $V$ contains functions that do not vanish at infinity. We will employ the Banach space $E$ defined by $E=V \times H^{1}(R) \times V$

For any $X=(\zeta, U, H) \in E$, the norm on $E$ is given by $\|X\|_{E}=\|\zeta\|_{V}+\|U\|_{H^{1}(R)}+\|H\|_{V}$

The following lemma gives the Lipschitz bounds we need on $Q$ and $P$.

Lemma 3.1. For any $X=(\zeta, U, H) \in E$, we define the maps $Q$ and $P$ as $Q(X)=Q$ and $P(X)=P$ where $Q$ and $P$ are given by (2.8) and (2.9), respectively. Then, $P$ and $Q$ are Lipschitz maps on bounded sets from $E$ $H^{1}(R)$.

Moreover, we have

$$
Q_{\xi}=-\frac{1}{2} H_{\xi}-\left(\frac{1}{2} U^{2}-P\right)\left(1+\zeta_{\xi}\right) \text { and } P_{\xi}=Q\left(1+\zeta_{\xi}\right)
$$

Note: The proof is not given in this article

Let $R_{1}: E \rightarrow V$ and $: R_{2}: E \rightarrow H^{1}(R)$, be two B-Lipschitz maps. Then, the product $X \rightarrow R_{1}(X) R_{2}(X)$ is also a B-Lipschitz map from $E$ to $H^{1}(R)$, or from $E$ to $V$.

Since the mapping $X: E \rightarrow e^{\zeta}$ is $B$-Lipschitz from $E$ to $V$, then $Q_{1}$ is the product of two B-Lipschitz maps, one from $E$ to $H^{1}(R)$ and the other from $E$ to $V$, it is B-Lipschitz map from $E$ to $\left.H^{1}(R)\right)$. Similarly, one proves that $Q_{2}$ is $B$-Lipschitz and therefore $Q$ is $B$-Lipschitz. Furthermore, $P$ is $B$-Lipschitz. The formulas (2.13) are obtained by direct computation using the product rule, see [15].

In the next theorem we prove the existence of solution to the system (2.10) by using a construction argument. 
Theorem 3.2. Given $\bar{X}=(\bar{\zeta}, \bar{U}, \bar{H})$ in $E$, there exists a time $T$ depending only on $\|\bar{X}\|_{E}$ such that the system (2.10) admits a unique solution in $C^{1}([0 ; T], E)$ with initial data $\bar{X}$.

Lemma 3.3 Given initial condition $\bar{X}=(\bar{U} ; \bar{H} ; \bar{\zeta}) \in E \cap\left[\mathrm{W}^{1, \infty}(\mathrm{R})\right]^{3}$, we consider the solution $X=$ $(\zeta ; U ; H) \in C^{1}([0 ; T] ; E)$ of (2.15) given by Theorem 3.3. Then, $X \in C^{1}\left([0 ; T], E \cap\left[\mathrm{W}^{1, \infty}(\mathrm{R})\right]^{3}\right.$.

Thus, this lemma allows us to pick up a special representative for $\left(\zeta_{\xi}, U_{\xi}, H_{\xi}\right)$ given by $(\alpha, \beta, \gamma)$, which is defined for all $\xi \in R$ and which, for any given $\xi$ satisfies the ordinary differential equation (2.15).

Proposition 3.4. Let $\mathrm{R}$ be a bounded linear operator on a Banach space $X$ into a Banach space . Let $f$ be in $C([0, T] ; X)$. Then, $R_{f}$ belongs to $C([0, T], Y)$ and therefore is Riemann integrable, and

$$
\int_{[0, T]} R f(t) d t=R \int_{[0, T]} f(t) d t=R f(t) d t
$$

Definition 3.5. The set $G$ is composed of all $(\zeta ; U ; H) \in E$ such that

$(\zeta ; U ; H) \epsilon\left[\mathrm{W}^{1, \infty}(\mathrm{R})\right]^{3}$

$y_{\xi} \geq 0 ; H_{\xi} \geq 0 ; y_{\xi}+H_{\xi}>0$ almost everywhere, and $\lim _{\xi \rightarrow 1} H(\xi)=0$

$y_{\xi} H_{\xi}=y_{\xi}^{2} \mathrm{U}^{2}+U_{\xi}^{2}$ almost everywhere

where we denote $y(\xi)=\zeta(\xi)+\xi$.

Note that if all functions are smooth and $y_{\xi}>0$, for initial data in $\mathrm{G}$, the solution of (2.10) exists globally.

Proof of the Theorem 3.2: First, we can rewritten the solution of (2.10) as follow,

$$
X(t)=\bar{X}+\int_{0}^{t} F(X(\mu)) d \mu
$$

where $F: E \rightarrow E$ is given by $F(X)=\left(U,-Q(X), U^{3}-2 P(X) U\right)$ where $X=(\zeta, U, H)$. The integrals are defined as Riemann integrals of continuous functions on the Banach space $E$. Using Lemma 3.1, we can check that each component of $F(X)$ is a product of functions that satisfy one of the assumptions of Lemma 3.3 and using this same lemma, we obtain that $F(X)$ is a Lipchitz function on any bounded set of $E$. Since $E$ is a Banach space, we use the standard contraction argument to prove the theorem.

We now turn to the proof of existence of global solutions of (2.10). We are interested in a particular class of initial data. In particular, we will only consider initial data that belong to $E \cap\left[\mathrm{W}^{1, \infty}(\mathrm{R})\right]^{3}$ where $\mathrm{W}^{1, \infty}(\mathrm{R})=\left\{\mathrm{f} \in \mathrm{C}_{\mathrm{b}}(\mathrm{R}) \mid \mathrm{f}_{\xi} \in \mathrm{L}^{\infty}(\mathrm{R})\right\}$. Given $(\bar{\zeta}, \bar{U}, \bar{H}) \in E \cap\left[\mathrm{W}^{1, \infty}(\mathrm{R})\right]^{3}$, we consider the short-time solution $(\zeta, U, H) \in \mathrm{C}([0, \mathrm{~T}], \mathrm{E})$ 2of (2.10) given by Theorem 3.2. Using the fact that $Q$ and $\mathrm{P}$ are Lipschitz on bounded sets (Lemma 3.1) and, since $\mathrm{X} 2 \mathrm{C}([0 ; \mathrm{T}]$; E), we can prove that $\mathrm{P}$ and $Q$ belongs to $\mathrm{C}\left([0, \mathrm{~T}], H^{1}(\mathrm{R})\right.$. We now consider $U, P$ and $Q$ as given function in $\mathrm{C}\left([0, \mathrm{~T}], H^{1}(\mathrm{R})\right.$. Then, for any fixed $\xi \epsilon \mathrm{R}$, we can solve the system of ordinary differential equations 
$\left\{\begin{array}{c}\frac{d}{d t} \alpha(t, \xi)=\beta(t, \xi) \\ \frac{d}{d t} \beta(t, \xi)=\frac{1}{2} \gamma(t, \xi)+\left(\left(\frac{1}{2} U^{2}-P\right)(t, \xi)\right)(1+\alpha(t, \xi)) \\ \frac{d}{d t} \gamma(t, \xi)=-((2 Q U)(t, \xi))(1+\alpha(t, \xi))+\left(\left(3 U^{2}-2 P\right)(t, \xi)\right) \beta(t, \xi)\end{array}\right.$

which is obtained by substituting $\zeta_{\xi}, U_{\xi}$ and $H_{\xi}$ in (2.12) by the unknowns $\alpha, \beta$ and $\gamma$, respectively.

Notation: We have to specify the initial conditions for (2.15). Let A be the following set

$$
A=\left\{\xi \in R|| \bar{U}_{\xi}(\xi)\left|\leq\left\|\bar{U}_{\xi}\right\|_{L^{\infty}(R)} ;\right| \bar{H}_{\xi}(\xi)\left|\leq\left\|H_{\xi}\right\|_{L^{\infty}(R)} ;\right| \bar{\zeta}_{\xi}(\xi) \mid \leq\left\|\bar{\zeta}_{\xi}\right\|_{L^{\infty}(R)}\right\}
$$

We have that $A$ has full measure, that is, measure $\left(\left(A^{c}\right)=0\right.$. For $\xi \epsilon A$ we define $(\alpha(0 ; \xi) ; \beta(0 ; \xi) ; \gamma(0 ; \xi))=\left(\bar{U}_{\xi}(\xi) ; \bar{H}_{\xi}(\xi) ; \bar{\zeta}_{\xi}(\xi)\right)$. However, for $\xi \epsilon A^{c}$ we take $(\alpha(0 ; \xi) ; \beta(0 ; \xi) ; \gamma(0 ; \xi))=(0 ; 0 ; 0)$.

\section{References}

[1] A. Bressan and A. Constantin. Global conservative solutions of the Camassa-Holm equation. Preprint. Submitted, 2005.

[2] Constantin A., Escher J.: Global weak sols for a shallow water eq. Indiana Univ. Math...47:15001511(1998)

[3] A. Degasperis, D. Holm, A. N.W. Hone: Integrable and non-integrable equations with peakons. Theory and Experiment, vol. II, Gallipoli,2002. World Scientific, River Edge, NJ.(2003)

[4] R. Camassa and D. D. Holm. An integrable shallow water equation with peaked solitons.Phys. Rev. Lett., 71(11):1661\{1664, 1993.

[5] R. Camassa, D. D. Holm, and J. Hyman. A new integrable shallow water equation. Adv.Appl. Mech.,33, 1994.

[6] E. Wahl'en. On the peakon-antipeakon interaction. Dyn. Contin. Discrete Impuls. Syst.Ser. A Math. Anal

[7] R. S. Johnson. Camassa\{Holm, Korteweg-de Vries and related models for water waves. J.Fluid Mech., 455:63-82, 2002.

[8] A. Constantin and J. Escher. Global existence and blow-up for a shallow water equation.Ann. Scuola Norm. Sup. Pisa Cl. Sci. (4), 26(2):303\{328, 1998.

[9] A. Constantin and B. Kolev. On the geometric approach to the motion of inertial mechanical systems. J. Phys. A, 35(32):R51\{R79, 2002.

[10] A. Constantin and L. Molinet. Global weak solutions for a shallow water equation. Comm.Math. Phys., 211(1):45\{61, 2000.

[11] M. Fonte. Conservative solution of the Camassa Holm equation on the real linearXiv:math.AP/0511549,3 2005.

[12] H. Holden and X. Raynaud. Global multipeakon solutions of the Camassa-Holm equation. J. Hyperbolic Differ. Equ. to appear, 2006. paper IV in this thesis.

[13] G. M. Coclite, and H. Holden, and K. H, Karlsen. Well-posedness for a parabolic-elliptic system. Discrete Cont. Dynam. Systems 13:659\{682, 2005.

[14] G. M. Coclite, and H. Holden, and K. H, Karlsen. Global weak solutions to a generalized hyperelastic-rod wave equation. SIAM J. Math. Anal., 37:1044\{1069, 2005.

[15] L. C. Evans and R. F. Gariepy. Measure Theory and Fine Properties of Functions. CRC Press, Boca Raton, Florida, USA, 1992. 